$\underline{\text { Preprint typeset in JHEP style. - PAPER VERSION }}$

DAMTP-2002-60

hep-th/0205291

\title{
Penrose Limits of the Baryonic D5-brane
}

\author{
David Mateos and Selena Ng \\ Department of Applied Mathematics and Theoretical Physics \\ Centre for Mathematical Sciences \\ Wilberforce Road, Cambridge CB3 OWA, United Kingdom \\ E-mail: D.Mateos@damtp.cam.ac.uk, S.K.L.Ng@damtp.cam.ac.uk
}

\begin{abstract}
The Penrose limits of a D5-brane wrapped on the sphere of $A d S_{5} \times S^{5}$ and connected to the boundary by $N$ fundamental strings, which is dual to the baryon vertex of the $\mathcal{N}=4 S U(N)$ super Yang-Mills theory, are investigated. It is shown that, for null geodesics that lead to the maximally supersymmetric Hpp-wave background, the resulting D5-brane is a $1 / 2$-supersymmetric null brane. For an appropriate choice of radial geodesic, however, the limiting configuration is $1 / 4$-supersymmetric and closely related to the Penrose limit of a flat space BIon.
\end{abstract}

Keywords: Penrose Limit and pp-wave background, D-branes, Supersymmetry and Duality. 


\section{Contents}

$\begin{array}{ll}\text { 1. Introduction } & 1\end{array}$

2. Baryonic D5-branes 3

3. Null D5-branes in Hpp-waves 4

4. Radial Geodesics and D5-brane BIons 10

$\begin{array}{ll}\text { 5. Discussion } & 13\end{array}$

A. Penrose Limit of the Minkowski Space BIon $\quad 14$

\section{Introduction}

The Penrose limit of $A d S_{5} \times S^{5}$ with $N$ units of Ramond-Ramond (RR) five-form flux is either the maximally supersymmetric type IIB Hpp-wave [1] or ten-dimensional Minkowski space, depending on whether or not the vector tangent to the null geodesic along which the limit is taken possesses a component along the five-sphere [2]. The perturbative spectrum of the type IIB superstring is exactly computable both in flat space [3] and in the Hpp-wave background [4]. The precise identification of the sector of the dual $\mathcal{N}=4 S U(N)$ super Yang-Mills (SYM) theory that is selected by the Penrose limit allowed the authors of [5] to reproduce these perturbative string spectra from the large- $N$ limit of the gauge theory.

According to the AdS/CFT correspondence in its strongest form, the $\mathcal{N}=4 \mathrm{SYM}$ theory encodes the entire non-perturbative type IIB string theory in $A d S_{5} \times S^{5}$. In particular, D-brane states in $A d S_{5} \times S^{5}$, and their excitations, should be 'contained' within the field theory (possibly in the presence of external sources or defects). Examples of such states include D3-brane giant gravitons [6], baryonic D5-branes [7], and D5-branes embedded as $A d S_{4} \times S^{2}$ submanifolds (and their generalizations [8]) relevant to the AdS/dCFT correspondence [9]. An exact description of these states and their excitations in $A d S_{5} \times S^{5}$ is of course difficult because of the well-known problems for the quantization of strings in this background. Since this difficulty is absent once the 
Penrose limit is taken, one might expect that in this limit the above D-brane states become exactly tractable. This expectation has been recently realized for the D3-brane giant gravitons [10] and for the $A d S \times S$ D-branes [8]. The purpose of this paper is to determine the Penrose limit of the baryonic D5-brane.

In terms of string theory in $A d S_{5} \times S^{5}$, a static external 'quark' in the SYM theory (that is, a static external electric charge transforming in the fundamental representation of $S U(N)$ ) is represented by the endpoint of a fundamental string that terminates at the $A d S$ boundary $^{1}[11,12]$. Similarly, a static baryon vertex, namely, a static gaugeinvariant antisymmetric combination of $N$ external electric charges, is represented by a D5-brane wrapped on $S^{5}$ and with constant position in a constant-time slice of $A d S_{5}$, and connected to the $A d S_{5}$ boundary by $N$ fundamental strings [7]. The reason for this is that the flux of the background RR five-form through the $S^{5}$ sources the Born-Infeld (BI) $U(1)$ gauge field on the D5-brane, inducing $N$ units of electric charge. Since the D5 worldspace is compact, the total net charge must vanish. The extra contribution is provided by the endpoints of $N$ fundamental strings (with suitable orientations) connecting the D5-brane to the $A d S$ boundary. The antisymmetry of the baryon vertex follows from the fermionic nature of the ground state of each string.

Each string connecting the D5-brane to the $A d S$ boundary defines a 'direction' in $A d S_{5} \times S^{5}$. Since the supersymmetries preserved by a string depend on its orientation, one might expect that a generic baryon vertex will break supersymmetry completely. On the other hand, it might be suspected (correctly) that the most symmetric configuration, in which all strings start at the same point on the D5-brane and end at the same point on the $A d S$ boundary, preserves some fraction of supersymmetry. This is a very natural configuration to consider also from the gauge theory viewpoint, since it corresponds to a 'localized' baryon vertex, that is, one for which all the $N$ external quarks lie at the same point. Despite its higher degree of symmetry, the description of this configuration in terms of a perfectly spherical D5-brane is oversimplified because it ignores (at least) two essential effects caused by the coincidence of all the endpoints of the strings on the D5: ${ }^{2}$ the deformation of the shape of the D5-brane by the tension of the strings, and the highly inhomogeneous BI electric field on the D5-brane sourced by their endpoints.

Fortunately, these two effects are captured by the Dirac-Born-Infeld (DBI) action of the D5-brane coupled to the $A d S_{5} \times S^{5}$ background [13, 16, 17, 18]. In this description, moreover, the strings do not need to be treated separately, but instead arise as a 'spike' deformation of the D5-brane worldvolume; in this sense, this configuration provides an

\footnotetext{
${ }^{1} \mathrm{~A}$ dyon with electric and magnetic charges $p$ and $q$, respectively, is represented by the endpoint of a $(p, q)$ string.

${ }^{2}$ As was realized [13] after some early computations of the baryon mass [14, 15].
} 
AdS analog of the flat space BIon [19, 20]. Since all the strings end at the same point on the D5-brane, an $S O(5)$ subgroup of the $S O(6)$ isometry group of the five-sphere is preserved. It follows that the deformation of the D5-brane can be specified by giving the radial position of the brane in $A d S_{5}$ as a function $r(\theta)$ of the colatitude angle $\theta$ on the $S^{5}$, measured with respect to the endpoint of the strings. A condition on the function $r(\theta)$ for preservation of some supersymmetry was first found in [13]. An explicit solution of this condition, which is given in (2.3), was presented in [16]. It was subsequently shown that this solution saturates an energy bound and hence minimizes the energy for a fixed value of a topological charge [17]. Finally, it was verified that the fraction of preserved supersymmetry is $1 / 4$ by making use of the $\kappa$-symmetry transformations of the D5-brane [18].

Since the $S O(5)$-symmetric baryonic D5-brane completely wraps an $S^{4}$ inside the $S^{5}$, one might expect that the Penrose limit along an appropriate null geodesic tangent to the sphere would lead to a D5-brane extending along both light-cone directions $x^{ \pm}$ (as well as four other spacelike directions) in the resulting Hpp-wave spacetime. We will show, however, that this is not the case, the reason being that there is no null geodesic contained within the D5-brane worldvolume. Consequently, in the Penrose limit the brane is always 'pushed' to lie at constant $x^{+}$in the metric (3.1); this implies that it becomes a null brane, by which we mean a brane whose worldvolume is a null hypersurface ${ }^{3}$.

We will also consider the Penrose limit along null geodesics in the AdS radial direction. For generic geodesics of this type, the resulting configuration is a null flat D5-brane in Minkowski space. For the radial geodesic along the strings attached to the D5-brane, however, the Penrose limit is closely related to that of the flat space BIon.

\section{Baryonic D5-branes}

We begin by briefly reviewing the relevant results from $[13,16,17,18]$; we refer the reader to these references for details.

We write the metric on $A d S_{5} \times S^{5}$ as

$$
d s^{2}=r^{2}\left(-d t^{2}+\boldsymbol{d} \boldsymbol{x}^{2}\right)+\frac{d r^{2}}{r^{2}}+d \theta^{2}+\sin ^{2} \theta d \Omega_{4}^{2},
$$

where $d \Omega_{4}^{2}$ is the line element on a unit four-sphere and $\boldsymbol{x}$ are Cartesian coordinates on $\mathbb{E}^{3}$. The baryonic D5-brane is wrapped on the $S^{4}$ and lies at rest at a point in $\mathbb{E}^{3}$;

\footnotetext{
${ }^{3}$ This implies $\operatorname{det} g=0$, where $g$ is the induced metric on the brane; some authors use the term 'null brane' to refer instead to branes for which $\operatorname{det}(g+F)=0$, where $F$ is the BI field strength.
} 
by translational invariance we take it to be

$$
\boldsymbol{x}=0 \text {. }
$$

The radial position $r$ of the D5-brane in $A d S_{5}$ is then specified [16] as a function of the colatitude angle $\theta$ by

$$
r(\theta)=A f(\theta), \quad f(\theta)=\frac{(\pi-\theta+\sin \theta \cos \theta)^{1 / 3}}{\sin \theta},
$$

where $A$ is an arbitrary constant that sets the overall scale of the solution; the freedom of changing $A$ arises from the conformal invariance of the $A d S$ background. The profile $r(\theta)$ reflects the finite deformation of the shape of the D5-brane caused by the tension of the strings; in particular, $r(\theta)$ diverges as $r(\theta) \sim \theta^{-1}$ for $\theta \rightarrow 0$. This 'spike' singularity represents the $N$ fundamental strings connecting the D5-brane to the $A d S$ boundary at $r \rightarrow \infty$.

Note that we are only considering here the case where the integration constant $\nu$ of [16] is set to zero, since it is this case which genuinely corresponds to the baryon vertex in the dual $\mathcal{N}=4 S U(N)$ gauge theory; the physical interpretation of the solutions with $\nu \neq 0$ is rather unclear [16].

In addition to deforming the brane, the endpoints of the strings source the electric components of the worldvolume BI two-form, which thus takes the form $[13,16]$

$$
F=A E(\theta) d t \wedge d \theta
$$

where

$$
E(\theta)=g(\theta) \sqrt{\frac{f^{2}(\theta)+f^{\prime 2}(\theta)}{g^{2}(\theta)+\sin ^{8}(\theta)}}, \quad g(\theta)=\frac{3}{2}(\pi-\theta+\sin \theta \cos \theta)+\sin ^{3} \theta \cos \theta .
$$

Note that $F$ is $S O(5)$-invariant.

\section{Null D5-branes in Hpp-waves}

The Penrose limit of any spacetime is a pp-wave spacetime [21, 22] whose metric in Brinkman coordinates takes the form

$$
d s^{2}=-4 d x^{+} d x^{-}+A_{i j}\left(x^{+}\right) z^{i} z^{j}\left(d x^{+}\right)^{2}+d s^{2}\left(\mathbb{E}^{8}\right),
$$

where $z^{i}$ are Cartesian coordinates on $\mathbb{E}^{8}$. The maximally supersymmetric Hpp-wave

solution of type IIB supergravity corresponds to the particular case $A_{i j}=-\delta_{i j}$; there is also a RR five-form given by

$$
F_{5}=4 d x^{+} \wedge\left(d z^{1} \wedge \ldots \wedge d z^{4}+d z^{5} \wedge \ldots \wedge d z^{8}\right) .
$$


The Hpp-wave solution can be obtained as the Penrose limit of the $A d S_{5} \times S^{5}$ solution along a null geodesic whose tangent vector has a non-zero projection onto $S^{5}[2]$; following [23], we shall refer to such geodesics as 'generic null geodesics'.

In this section we will show that the Penrose limit of the baryonic D5-brane along any generic null geodesic is necessarily a null D5-brane, that is, a D5-brane whose worldvolume is a null six-dimensional hypersurface in the above metric. This follows from the general fact mentioned in the Introduction: a brane is always 'pushed' to lie at constant $x^{+}$in the pp-wave spacetime when the Penrose limit is taken along a null geodesic that intersects, but that is not contained within, its worldvolume. Although this result might be intuitive, we provide here a somewhat formal justification.

In the neighbourhood of a segment of a null geodesic $\gamma$ containing no conjugate points there exist local coordinates $\left\{U, V, Y^{i}\right\}$ such that the metric takes the form $[21,22,23]$

$$
d s^{2}=2 d V\left(d U+\alpha d V+\beta_{i} d Y^{i}\right)+C_{i j} d Y^{i} d Y^{j},
$$

where $\alpha, \beta_{i}$ and $C_{i j}$ are functions of all the coordinates. The geodesic $\gamma$ lies at $V=$ $Y^{i}=0$ and is affinely parametrized by $U$. The resulting metric $\bar{d} s^{2}$ in the Penrose limit along $\gamma$ may be obtained by rescaling the coordinates as

$$
U=u, \quad V=\Omega^{2} v, \quad Y^{i}=\Omega y^{i}
$$

for positive real constant $\Omega$, and computing the limit

$$
\overline{d s}^{2} \equiv \lim _{\Omega \rightarrow 0} \Omega^{-2} d s^{2}
$$

while keeping $u, v$ and $y^{i}$ fixed. It can then be brought to the form (3.1) by a further change of variables that, in particular, identifies $u=x^{+}$.

Consider now a brane embedded with $(p+1)$-dimensional worldvolume $\mathcal{W}$ in the spacetime in question. Unless $\gamma$ intersects $\mathcal{W},{ }^{4}$ the brane 'disappears' upon taking the Penrose limit, because only a region infinitesimally close to $\gamma$ (which is magnified by an 'infinite' amount) survives the limit [23]. Let us therefore focus on the other two possibilities, that is, either $\gamma$ intersects $\mathcal{W}$ at an isolated point $q=\left(U_{0}, 0,0\right)$ or $\gamma$ is actually contained within $\mathcal{W}^{5}{ }^{5}$ In both cases, in a neighbourhood of $q$ the brane embedding may be specified in implicit form as

$$
f^{k}\left(U, V, Y^{i}\right)=0
$$

\footnotetext{
${ }^{4}$ Or becomes arbitrarily close to it asymptotically; see next section.

${ }^{5}$ This is a local analysis; globally, of course, $\gamma$ might intersect $\mathcal{W}$ at more than one point and/or have a number of segments contained within it.
} 
for functions $f^{k}, k=0, \ldots, p$. After the rescaling (3.4) we can expand these functions as

$$
f^{k}\left(U, V, Y^{i}\right)=f^{k}(u, 0,0)+\Omega^{2} f_{V}^{k}(u, 0,0) v+\Omega f_{i}^{k}(u, 0,0) y^{i}+\cdots,
$$

where $f_{V}^{k}=\partial_{V} f^{k}$ and $f_{i}^{k}=\partial_{Y^{i}} f^{k}$. Since $\gamma$ lies at $V=Y^{i}=0$, if $\gamma$ is contained within $\mathcal{W}$ then the first term in this expansion vanishes identically for all $u$, and hence in the limit $\Omega \rightarrow 0$ equations (3.6) determine a subset of the $v$ and $y^{i}$ coordinates as functions of the remaining ones and/or of $u$. Instead, if $\gamma$ is not contained in $\mathcal{W}$ then in the limit $\Omega \rightarrow 0$ the above equations imply $f^{k}(u, 0,0)=0$, and hence that the brane lies at $u=U_{0}$ or, equivalently, at constant $x^{+}$in Brinkman coordinates.

We now return to the baryonic D5-brane. Since we wish to prove that there is no generic null geodesic contained within the D5-brane worldvolume, we need only consider geodesics that lie at $\boldsymbol{x}=0$. If we view $S^{5}$ as embedded in $\mathbb{R}^{6}$ in the usual way, then the projection on $S^{5}$ of any generic null geodesic is a circle of unit radius on some two-plane. In the three-dimensional subspace of $\mathbb{R}^{6}$ generated by this plane and the axis with respect to which the azimuthal angle $\theta$ is measured $^{6}$ we may define new spherical coordinates $\Theta, \Phi$ such that the geodesic lies at $\Phi=0$ and is parametrized by the new azimuthal angle $\Theta$ (see Figure 1). In terms of these new coordinates we have

$$
\theta=h(\Theta, \Phi) \equiv \arccos (\sin \xi \cos \Theta-\cos \xi \sin \Theta \sin \Phi)
$$

where $\xi$ is the angle between the normal to the plane and the $\theta$-axis. The radial position in $A d S_{5}$ of the geodesic is given in terms of the new azimuthal angle by $r=\ell^{-1} \sin \Theta$, where $\ell$ is the angular momentum of the geodesic [23]. It follows that the condition for a point to belong both to the geodesic and to the D5-brane worldvolume is

$$
\ell^{-1} \sin \Theta=A f(h(\Theta, 0)) .
$$

Since this equation has at most two solutions (which occurs for appropriate values of $A, \ell$ and $\xi$ ), we conclude that there is no generic null geodesic contained within the D5-brane worldvolume, and hence that the Penrose limit along any such geodesic leads to a null D5-brane in the IIB Hpp-wave background.

In order to determine the resulting D5-brane more precisely, we first change coordinates from $\{t, r, \Theta\}$ to $\{U, V, \Psi\}$ adapted to the geodesic and defined by [23]

$$
t=-\ell \cot \ell U-V+\ell \Psi, \quad r=\ell^{-1} \sin \ell U, \quad \Theta=\ell U+\Psi
$$

The geodesic lies at $V=\Psi=\boldsymbol{x}=0$, so we further rescale the coordinates as

$$
U=u, \quad V=\Omega^{2} v, \quad \boldsymbol{x}=\Omega \tilde{\boldsymbol{x}}, \quad \Psi=\Omega \psi, \quad \Phi=\Omega y
$$

\footnotetext{
${ }^{6}$ If this axis is contained within the two-plane it suffices to set $\Theta=\theta$ in the discussion below.
} 


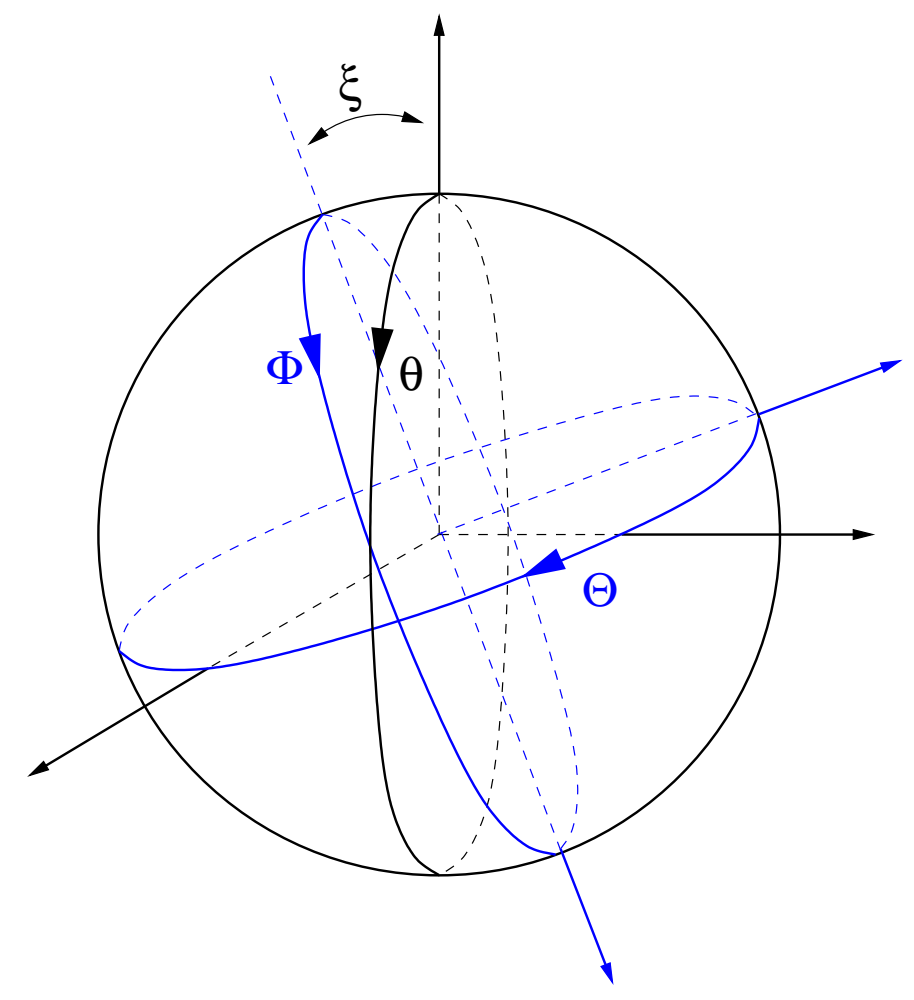

Figure 1: New coordinates on the five-sphere.

and take the limit (3.5) with the result

$$
\overline{d s}^{2}=2 d u d v+\cos ^{2} \ell u d \psi^{2}+\ell^{-2} \sin ^{2} \ell u d \tilde{\boldsymbol{x}}^{2}+\sin ^{2} \ell u d \boldsymbol{y}^{2},
$$

where we have set $y=|\boldsymbol{y}|$ for Cartesian coordinates $\boldsymbol{y}$ on $\mathbb{E}^{4}$. This is just the Hpp-wave metric in Rosen coordinates. The final transformation

$$
\begin{aligned}
& u=x^{+}, \quad \psi=z^{1} \sec \ell x^{+}, \quad \tilde{\boldsymbol{x}}=\ell^{2} \tilde{\boldsymbol{z}} \csc \ell x^{+}, \quad \boldsymbol{y}=\boldsymbol{z} \csc \ell x^{+}, \\
& v=2 x^{-}-\frac{\ell}{2} \tan \ell x^{+}\left(z^{1}\right)^{2}+\frac{\ell}{2} \cot \ell x^{+}\left(\tilde{\boldsymbol{z}}^{2}+\boldsymbol{z}^{2}\right)
\end{aligned}
$$

brings the metric to the form (3.1) with $A_{i j}=-\delta_{i j}, \tilde{\boldsymbol{z}}=\left(z^{2}, z^{3}, z^{4}\right)$ and $\boldsymbol{z}=\left(z^{5}, z^{6}, z^{7}, z^{8}\right)$.

The original embedding equation (2.2) becomes simply $\tilde{\boldsymbol{z}}=0$ in the final coordinates, whereas (2.3) yields (3.9) with $\Theta$ replaced by $\ell x^{+}$, which implies $x^{+}=$constant; ${ }^{7}$ this means that the brane extends along the directions $x^{-}, z^{1}$ and $\boldsymbol{z}$. The limiting BI field $\bar{F}$ is similarly determined by first performing the above changes of coordinates

\footnotetext{
${ }^{7}$ If equation (3.9) has two solutions then the Penrose limit results in two parallel branes at two different values of $x^{+}$.
} 
and rescalings and then computing the limit

$$
\bar{F}=\lim _{\Omega \rightarrow 0} \Omega^{-2} F .
$$

The result is

$$
\bar{F}=B d z^{1} \wedge d z
$$

where $z=|\boldsymbol{z}|$ and $B$ is a constant that can be determined explicitly but whose precise value will not be needed in the following. Note that the original electric field gives rise to a magnetic field after the Penrose limit; this can presumably be understood as the magnetic field seen by a Lorentz-transformed observer whose worldline approaches the null geodesic.

As anticipated, we have obtained a null D5-brane in the sense that $\operatorname{det} g=0$, where $g$ is the induced metric on the brane. Since we also have

$$
\operatorname{det}(g+F)=0
$$

the appropriate action to describe the dynamics of this configuration is not the ordinary DBI action but rather $^{8}[24,25]$

$$
S=\int \frac{1}{2 e} \operatorname{det}(g+F),
$$

where $e$ is an independent worldvolume density whose equation of motion enforces the condition (3.16). We have verified that the remaining field equations derived from (3.17) are also satisfied (with constant $e$ ); this should be expected given that the original configuration solved the D5-brane equations in $A d S_{5} \times S^{5}$. Moreover, since the original solution is supersymmetric, so should be the resulting configuration after the Penrose limit, as we now proceed to show.

The supersymmetries preserved by a D5-brane are those generated by Killing spinors $\epsilon$ of the background that satisfy [26]

$$
\Xi \epsilon=\sqrt{-\operatorname{det}(g+F)} \epsilon,
$$

where $\Xi$ is the matrix occurring in the kappa-symmetry transformations of the fermions in the supersymmetric extension of the action (3.17) [25]; by construction, $\Xi^{2}=-\operatorname{det}(g+F)$, so in the present case $\Xi$ is nilpotent.

The background metric (3.1) can be written as

$$
d s_{10}^{2}=2 e^{+} e^{-}+e^{i} e^{i}
$$

\footnotetext{
${ }^{8}$ The coupling to the background RR five-form vanishes because of the D5-brane orientation.
} 
for orthonormal one-forms

$$
e^{-}=2 d x^{-}+\frac{z^{i} z^{i}}{2} d x^{+}, \quad e^{+}=-d x^{+}, \quad e^{i}=d z^{i} .
$$

In this basis the Killing spinors take the form $[1,2,8]$

$$
\epsilon=\left(1-\frac{i}{2} \Gamma_{-} M\right) \exp \left(-\frac{i}{2} x^{+} N\right) \epsilon_{0}
$$

where

$$
M=\sum_{a=1}^{4} z^{a} \Gamma_{a} \Gamma_{1234}+z^{a+4} \Gamma_{a+4} \Gamma_{5678}, \quad N=\Gamma_{1234}+\Gamma_{5678} .
$$

Here $\left\{\Gamma_{+}, \Gamma_{-}, \Gamma_{1}, \ldots, \Gamma_{8}\right\}$ are real tangent-space constant Dirac matrices associated to the basis (3.20), and $\epsilon_{0}$ is a complex 16-component constant spinor of negative chirality, that is, $\Gamma_{+-12345678} \epsilon_{0}=-\epsilon_{0}$.

For the configuration of interest here the kappa-symmetry matrix takes the form

$$
\Xi=2 \Gamma_{15678}\left(\Gamma_{1} \not B-K\right) I \Gamma_{-},
$$

where the operations $K$ and $I$ act on spinors as

$$
K \epsilon=\epsilon^{*}, \quad I \epsilon=-i \epsilon
$$

and where we have defined

$$
\not B=\sum_{a=5}^{8} B_{a} \Gamma^{a}, \quad B_{a}=\frac{z^{a}}{z} B .
$$

Equation (3.18) thus becomes

$$
2 \Gamma_{15678} \exp \left(-\frac{i}{2} x^{+} N\right)\left(\Gamma_{1} \not B-K\right) I \Gamma_{-} \epsilon_{0}=0,
$$

where we have made use of the fact that $\Gamma_{-}^{2}=0$. Since the matrix acting on $\Gamma_{-} \epsilon_{0}$ is invertible, we conclude that the only solutions to this equation are provided by spinors that satisfy

$$
\Gamma_{-} \epsilon_{0}=0 \text {. }
$$

This means that $1 / 2$ of the 32 supersymmetries of the Hpp-wave background are unbroken by the D5-brane, which is twice the amount of supercharges preserved by the original baryonic D5-brane. This enhancement of supersymmetry in the Penrose limit has been observed previously in [27, 28, 29]. Note that, unlike in [8], all the D5-brane configurations in the Hpp-wave background with the same orientation as the one we have obtained via the Penrose limit preserve the same supersymmetries, irrespectively of whether or not the values of $x^{+}$and $B$ are those particular ones dictated by the limit. 


\section{Radial Geodesics and D5-brane BIons}

In this section we shall examine the Penrose limit of the baryonic D5-brane along radial null geodesics, by which we mean null geodesics that lie at a point $p$ on $S^{5}$ and at $\boldsymbol{x}=0$ in $A d S_{5}$. If $p$ is not the North pole, that is, the point $\theta=0$, then the radial geodesic intersects the D5-brane at one point and the Penrose limit along it leads to a null D5-brane in Minkowski space; since this is similar to the case discussed in detail in the previous section, we shall not elaborate on this further. Instead, in this section we shall focus on the Penrose limit along the geodesic at $\theta=0$. Since this does not intersect the D5-brane, it might seem that the D5-brane disappears upon taking the limit. However, because this geodesic becomes arbitrarily close to a region of the brane worldvolume asymptotically (that is, as $r \rightarrow \infty$ ), this region survives the limit and leads to a D5-brane embedded non-trivially in Minkowski space.

The change of coordinates

$$
U=r, \quad V=t+r^{-1}
$$

brings the metric (2.1) to the form

$$
d s^{2}=2 d U d V-U^{2} d V^{2}+U^{2} \boldsymbol{d} \boldsymbol{x}^{2}+d \theta^{2}+\sin ^{2} \theta d \Omega_{4}^{2}
$$

The radial geodesic of interest lies at $V=\theta=\boldsymbol{x}=0$, and is parametrized by $U$. To take the Penrose limit we perform the usual rescaling

$$
U=u, \quad V=\Omega^{2} v, \quad \boldsymbol{x}=\Omega \tilde{\boldsymbol{x}}, \quad \theta=\Omega y
$$

and take the limit (3.5). The resulting metric is

$$
\overline{d s}^{2}=2 d u d v+u^{2} \boldsymbol{d} \tilde{\boldsymbol{x}}^{2}+\boldsymbol{d} \boldsymbol{y}^{2}
$$

where we have set $y=|\boldsymbol{y}|$ for Cartesian coordinates $\boldsymbol{y}$ on $\mathbb{E}^{5}$. The further coordinate transformation

$$
u=\frac{X+y^{0}}{2}, \quad v=\left(X-y^{0}\right)+\frac{\boldsymbol{w}^{2}}{X+y^{0}}, \quad \tilde{\boldsymbol{x}}=\frac{2 \boldsymbol{w}}{X+y^{0}}
$$

brings the metric to the form

$$
\overline{d s}^{2}=-\left(d y^{0}\right)^{2}+\boldsymbol{d} \boldsymbol{y}^{2}+d X^{2}+\boldsymbol{d} \boldsymbol{w}^{2},
$$

which, as expected [23], is just the Minkowski metric. 
The embedding equation (2.2) for the D5-brane becomes simply $\boldsymbol{w}=0$ in the new coordinates, while equation (2.3) yields

$$
X+y^{0}=2 A f(\Omega y)=\frac{2 \pi^{1 / 3} A}{\Omega y}+\mathcal{O}(\Omega) .
$$

We see that, because $r(\theta)$ diverges as $\theta \rightarrow 0$, in order for the limit $\Omega \rightarrow 0$ with finite $y^{0}$ and $X$ to exist we must rescale

$$
A=\frac{a}{2 \pi^{1 / 3}} \Omega
$$

for constant, $\Omega$-independent $a$ (the numerical factor is for later convenience). Note that we are allowed to do so because (2.3) yields a (supersymmetric) solution of the D5-brane equations of motion for any $A$. The rescaling above means that, as we focus on a region closer and closer to the geodesic, we also focus on a solution with smaller and smaller $A$. This procedure gives a finite limit for the embedding equation, namely

$$
X=\frac{a}{y}-y^{0} .
$$

Thus in the Penrose limit we may interpret $\left\{y^{0}, \boldsymbol{y}\right\}$ as worldvolume coordinates of the D5-brane and $X$ as the only excited transverse scalar with profile given by (4.9).

The limiting BI field strength on the D5-brane is obtained in an analogous manner by performing the coordinate tranformations and the rescalings above, and then computing the limit (3.14), for which it is useful to note that

$$
E(\Omega y)=\frac{\pi^{1 / 3}}{\Omega^{2} y^{2}}+\mathcal{O}(1) .
$$

The result is $\bar{F}=E_{a}(\boldsymbol{y}) d y^{0} \wedge d y^{a}$, where

$$
\boldsymbol{E}=\nabla X
$$

Note that precisely the same rescaling of $A$ required to obtain a well-defined limiting embedding also yields a finite BI field. The ultimate reason behind this is presumably supersymmetry, since this determines the original electric field in terms of the scalar transverse to the D5-brane (the radial position $r$ in $A d S_{5}$ ), as specified by (2.5). This non-linear equation simplifies in the Penrose limit to (4.11), which is precisely the condition for an ordinary Minkowski space BIon to preserve some fraction of supersymmetry $[19,20,30]$. In fact, the Penrose limit of the ordinary BIon along the direction of the string yields a transverse scalar

$$
X=\frac{a}{y^{3}}-y^{0}
$$


and an electric field given by (4.11) (see the Appendix for details). Note that the only difference is that in this case $X$ is a harmonic function on the D5-brane worldspace, as required by its equation of motion before the Penrose limit. It might seem surprising, therefore, that both configurations (4.9) and (4.12) are supersymmetric and solve the same D5-brane equations of motion, as one would expect from their origin as Penrose limits of supersymmetric solutions. We will return to this issue below.

Since in both cases

$$
\dot{X}=-1
$$

where $\dot{X} \equiv \partial_{0} X$, each point on the brane is moving in the $X$-direction at the speed of light. One might think that this is only possible for a brane whose induced metric $g$ is null in the sense that $\operatorname{det} g=0$. However, because a D-brane is boost-invariant, only the component of the velocity orthogonal to its surface is physical, and this ensures that the physical velocity of all points on the D5-brane is subluminal ${ }^{9}$. In particular, we find

$$
\operatorname{det} g=-1+\dot{X}^{2}-(\nabla X)^{2}
$$

and therefore the induced metric on the D5-brane is everywhere Lorentzian (although it does approach a null metric as $y \rightarrow \infty)$. However, a calculation reveals that

$$
\operatorname{det}(g+F)=-\left(1-\boldsymbol{E}^{2}\right)\left(1+(\boldsymbol{\nabla} X)^{2}\right)-(\boldsymbol{E} \cdot \boldsymbol{\nabla} X)^{2}+\dot{X}^{2}
$$

does vanish, and consequently that the appropriate action to describe the D5-brane dynamics is again (3.17). For configurations satisfying (4.11) and (4.13) the equations of motion obtained by varying this action with respect to $X$ and to the BI gauge field reduce to the unique condition

$$
\boldsymbol{\nabla} \cdot\left(e^{-1} \nabla X\right)=0 .
$$

We thus see that the two configurations (4.9) and (4.12) are particular cases of a more general class of solutions characterized by the fact that $X$ depends solely on the radial coordinate $y$ on the D5-brane worldspace; these solutions satisfy equation (4.16) with $e=y^{4} d X / d y$. This arbitrariness in the profile of the D5-brane is reminiscent of that recently discussed in the context of D2-brane supertubes [32].

We finally turn to showing that the conditions (4.11) and (4.13) guarantee not only that the D5-brane field equations are satisfied but also that $1 / 4$ supersymmetry is preserved. Again we must seek solutions of equation (3.18), where now $\epsilon$ is a constant 16-component complex Killing spinor of the Minkowski background and

$$
\Xi=\Gamma_{012345} K I\left(1-\dot{X} \Gamma_{0 X}\right)+\Gamma_{12345} \Gamma^{a} I\left(E_{a}-\partial_{a} X \Gamma_{0 X} K\right)+\Gamma_{12345 X} I E_{a} \partial_{b} X \Gamma^{a b},
$$

\footnotetext{
${ }^{9} \mathrm{An}$ analogous situation in the context of fundamental strings has been recently discussed in [31].
} 
where $a, b=1, \ldots, 5$ and, as in the previous section, the Dirac matrices appearing here are real flat-space constant matrices. It follows that if conditions (4.11) and (4.13) hold then equation (3.18) is verified by spinors subject to the constraints

$$
\Gamma_{0 X} K \epsilon=\epsilon, \quad \Gamma_{0 X} \epsilon=-\epsilon .
$$

The first condition is associated to fundamental strings along the $X$-direction, whereas the second one corresponds to a wave along the same direction. Since the two constraints are mutually compatible, $1 / 4$ supersymmetry is preserved. In the two particular cases discussed above, the strings in the $X$-direction arise of course from the strings originally attached to the D5-brane before the Penrose limit. Instead, the wave arises because in taking the limit the brane is effectively boosted to the speed of light in the $X$-direction, as discussed above. Note that there is no trace of a constraint associated to the presence of a D5-brane, again in analogy to the case of D2-brane supertubes [33].

\section{Discussion}

We have examined in detail the possible Penrose limits of the D5-brane dual to the baryon vertex of the $\mathcal{N}=4$ SYM theory. While the resulting background depends solely on whether or not the null geodesic along which the limit is taken possesses a non-zero projection on $S^{5}$, the limit of the baryon vertex depends on the relative orientation between the geodesic and the D5-brane.

For generic null geodesics, that is, for geodesics that lead to the maximally supersymmetric Hpp-wave solution, the resulting 1/2-supersymmetric D5-brane is always a null brane, in the sense that its worldvolume is a null-hypersurface. This situation should be contrasted with the Penrose limit of the D3-brane giant gravitons [10] and of the $A d S \times S$ D-branes [8]. These branes are static with respect to the AdS global time $^{10} \tau$ and wrap some great circle (or a higher-dimensional submanifold) within the $S^{5}$. For such types of branes there is always a null geodesic contained within their worldvolumes. To see this, write the metric on $A d S_{5} \times S^{5}$ as

$$
d s^{2}=-\cosh ^{2} \rho d \tau^{2}+d \rho^{2}+\sinh ^{2} \rho d \Omega_{3}^{2}+\cos ^{2} \phi d \psi^{2}+d \phi^{2}+\sin ^{2} \phi d \Omega_{3}^{2},
$$

where $\psi$ is the coordinate along the great circle. Then the curve $\tau=\psi, \rho=\phi=0$ is a null geodesic (of the type considered in [5]) that clearly lies within the worldvolume of the brane in question. The Penrose limit along this geodesic leads to a brane

\footnotetext{
${ }^{10}$ Although the giant gravitons with angular momentum $J<N$ are time-dependent configurations, the case $J=N$ considered in [10] corresponds to a static D3-brane whose angular momentum arises entirely from its Wess-Zumino coupling to the background RR five-form.
} 
that extends along both $x^{+}$and $x^{-}$(and possibly some other spatial directions) in the Hpp-wave metric (3.1), and therefore the induced metric possesses Lorentzian signature. In contrast, the baryonic D5-brane is static with respect to the Poincaré time $t$, and we have seen that in this case there is no generic null geodesic contained within its worldvolume; for geodesics that intersect it at a point, the D5-brane is 'pushed' to lie at constant $x^{+}$in the resulting pp-wave background. This result makes the physical interpretation of the baryonic D5-brane in the Penrose limit unclear, especially in relation to the $\mathcal{N}=4 \mathrm{SYM}$ theory. We leave the investigation of this point for the future.

We have also analyzed the Penrose limit along radial null geodesics that lie at a point $p$ on $S^{5}$. If $p$ is not the North pole, that is, the point at which the fundamental strings end on the D5-brane, then the resulting configuration is a null flat D5-brane in Minkowski space. If $p$ is the North pole then the radial geodesic does not intersect the D5-brane, so it might seem that the latter disappears upon taking the Penrose limit. However, we showed that if the overall size of the baryon vertex is appropriately rescaled then the limit results in a 1/4-supersymmetric D5-brane closely related to the Penrose limit of the flat space BIon.

Note added: While this paper was being type-written we received [34], where some issues related to those reported here are considered.

\section{Acknowledgments}

We are grateful to Prem Kumar for collaboration at earlier stages of this work, and to Michael B. Green for discussions. D.M. is supported by a PPARC fellowship. S.N. is supported by the British Federation of Women Graduates and the Australian Federation of University Women (Queensland).

\section{A. Penrose Limit of the Minkowski Space BIon}

The BIon $[19,20,30]$ is a solution of the equations of motion of a D-brane embedded in Minkowski space that represents a semi-infinite string ending orthogonally on the D-brane; it preserves $1 / 2$ of the D-brane worldvolume supersymmetry (that is, $1 / 4$ of the 32 Minkowski supersymmetries).

We write the Minkowski metric as

$$
d s^{2}=-d T^{2}+d \boldsymbol{Y}^{2}+d X^{2}+d \boldsymbol{Z}^{2}
$$


where $\boldsymbol{Y}$ and $\boldsymbol{Z}$ are Cartesian coordinates on $\mathbb{E}^{5}$ and $\mathbb{E}^{3}$, respectively. Suppose the D5brane lies at $\boldsymbol{Z}=0$ and that $X$ is the direction of the string. Any configuration such that $\boldsymbol{E}=\boldsymbol{\nabla} X$ and $\boldsymbol{\nabla}^{2} X=0$, where $\boldsymbol{E}$ is the BI electric field and $\boldsymbol{\nabla}$ is the gradient operator in $\mathbb{E}^{5}$, solves the D5-brane field equations and preserves $1 / 2$ supersymmetry. For spherically symmetric configurations we thus have

$$
X=\frac{A}{Y^{3}}
$$

where $A$ is a constant and $Y=|\boldsymbol{Y}|$. This represents a string ending on the brane at $Y=0$.

In order to take the Penrose limit along the null geodesic $T=X$ that runs along the string we define new coordinates

$$
U=T+X, \quad V=T-X
$$

perform the rescaling

$$
U=u, \quad V=\Omega^{2} v, \quad \boldsymbol{Y}=\Omega \boldsymbol{y}, \quad \boldsymbol{Z}=\Omega \boldsymbol{z}
$$

and take the limit (3.5). The resulting metric

$$
\overline{d s}^{2}=-d u d v+d \boldsymbol{y}^{2}+d \boldsymbol{z}^{2}
$$

is of course the Minkowski metric again. Equation (A.2) in the rescaled coordinates becomes

$$
u=\frac{2 A}{\Omega^{3} y^{3}}+\Omega^{2} v
$$

so we see that to achieve a finite limit we must rescale $2 A=\Omega^{3} a$ for constant $\Omega$ independent $a$, in which case we obtain

$$
x=\frac{a}{y^{3}}-t
$$

(where we have set $u=t+x$ and $v=t-x$ ) thus reproducing (4.12). The resulting electric field is similarly computed by performing the coordinate changes and rescalings above and taking the limit (3.14), with the expected result $\overline{\boldsymbol{E}}=\boldsymbol{\nabla} x$.

The configuration we have obtained can also be derived, as might be expected, by boosting the initial BIon along the negative $X$-direction to the speed of light while rescaling $A$ appropriately in order to achieve a finite limit. 


\section{References}

[1] M. Blau, J. Figueroa-O'Farrill, C. Hull and G. Papadopoulos, A New Maximally Supersymmetric Background of Type IIB Superstring Theory, J. High Energy Phys. 01 (2002) 047, hep-th/0110242.

[2] M. Blau, J. Figueroa-O'Farrill, C. Hull and G. Papadopoulos, Penrose Limits and Maximal Supersymmetry, hep-th/0201081.

[3] M. B. Green, J. H. Schwarz and E. Witten, Superstring Theory, Vols 1 \& 2, Cambridge University Press, 1987.

[4] R. R. Metsaev, Type IIB Green-Schwarz Superstring in Plane Wave Ramond-Ramond Background, Nucl. Phys. B 625 (2002) 70, hep-th/0112044.

[5] D. Berenstein, J. M. Maldacena and H. Nastase, Strings in Flat Space and pp-waves from $\mathcal{N}=4$ Super Yang-Mills, J. High Energy Phys. 04 (2002) 013, hep-th/0202021.

[6] J. McGreevy, L. Susskind and N. Toumbas, Invasion of the Giant Gravitons from Anti de Sitter Space, J. High Energy Phys. 06 (2000) 008, hep-th/0003075.

[7] E. Witten, Baryons and Branes in Anti de Sitter Space, J. High Energy Phys. 07 (1998) 006, hep-th/9805112.

[8] K. Skenderis and M. Taylor, Branes in AdS and pp-wave Spacetimes, hep-th/0204054.

[9] A. Karch and L. Randall, Open and Closed String Interpretation of SUSY CFT's on Branes with Boundaries, J. High Energy Phys. 06 (2001) 063, hep-th/0105132.

[10] V. Balasubramanian, M. Huang, T. S. Levi and A. Naqvi, Open Strings from $\mathcal{N}=4$ Super Yang-Mills, hep-th/0204196.

[11] S. Rey and J. Yee, Macroscopic Strings as Heavy Quarks: Large-N Gauge Theory and Anti de Sitter Supergravity, Eur. Phys. J. Direct. C 22 (2001) 379, hep-th/9803001.

[12] J. M. Maldacena, Wilson Loops in Large N Field Theories, Phys. Rev. Lett. 80 (1998) 4859 , hep-th/9803002.

[13] Y. Imamura, Supersymmetries and BPS Configurations on Anti-de Sitter Space, Nucl. Phys. B 537 (1999) 184, hep-th/9807179.

[14] A. Brandhuber, N. Itzhaki, J. Sonnenschein and S. Yankielowicz, Baryons from Supergravity, J. High Energy Phys. 07 (1998) 020, hep-th/9806158.

[15] Y. Imamura, Baryon Mass and Phase Transitions in Large N Gauge Theory, Prog. Theor. Phys. 100 (1998) 1263, hep-th/9806162. 
[16] C. G. Callan, A. Güijosa and K. G. Savvidy, Baryons and String Creation from the Fivebrane Worldvolume Action, Nucl. Phys. B 547 (1999) 127, hep-th/9810092.

[17] B. Craps, J. Gomis, D. Mateos and A. Van Proeyen, BPS Solutions of a D5-brane Worldvolume in a D3-brane Background from Superalgebras, J. High Energy Phys. 04 (1999) 004, hep-th/9901060.

[18] J. Gomis, A. Ramallo, J. Simón and P. K. Townsend, Supersymmetric Baryonic Branes, J. High Energy Phys. 11 (1999) 019, hep-th/9907022.

[19] C. G. Callan and J. M. Maldacena, Brane Dynamics From the Born-Infeld Action, Nucl. Phys. B 513 (1998) 198, hep-th/9708147.

[20] G. W. Gibbons, Born-Infeld Particles and Dirichlet p-branes, Nucl. Phys. B 514 (1998) 603, hep-th/9709027.

[21] R. Penrose, Any Spacetime Has a Plane-wave as a Limit, in 'Differential Geometry and Relativity', p. 271, Reidel, Dordrecht, 1976.

[22] R. Güven, Plane Wave Limits and T-Duality, Phys. Lett. B 482 (2000) 255, hep-th/0005061.

[23] M. Blau, J. Figueroa-O'Farrill and G. Papadopoulos, Penrose Limits, Supergravity and Brane Dynamics, hep-th/0202111.

[24] U. Lindström and R. von Unge, A Picture of D-branes at Strong Coupling, Phys. Lett. B 403 (1997) 233, hep-th/9704051.

[25] E. Bergshoeff and P. K. Townsend, Super D-branes Revisited, Nucl. Phys. B 531 (1998) 226 , hep-th/9804011.

[26] E. Bergshoeff, R. Kallosh, T. Ortín and G. Papadopoulos, Kappa-symmetry, supersymmetry and intersecting branes, Nucl. Phys. B 502 (1997) 149, hep-th/9705040.

[27] N. Itzhaki, I. R. Klebanov and S. Mukhi, PP Wave Limit and Enhanced Supersymmetry in Gauge Theories, J. High Energy Phys. 03 (2002) 048, hep-th/0202153.

[28] J. Gomis and H. Ooguri, Penrose Limit of N=1 Gauge Theories, hep-th/0202157.

[29] L. A. Pando Zayas and J. Sonnenschein, On Penrose Limits and Gauge Theories, hep-th/0202186.

[30] J. P. Gauntlett, J. Gomis and P. K. Townsend, BPS Bounds for Worldvolume Branes, J. High Energy Phys. 01 (1998) 003, hep-th/9711205.

[31] D. Mateos, S. Ng and P. K. Townsend, Supercurves, hep-th/0204062, to be published in Phys. Lett. B. 
[32] D. Mateos, S. Ng and P. K. Townsend, Tachyons, Supertubes and Brane/Anti-Brane Systems, J. High Energy Phys. 03 (2002) 016, hep-th/0112054.

[33] D. Mateos and P. K. Townsend, Supertubes, Phys. Rev. Lett. 87 (2001) 011602, hep-th/0103030.

[34] S. Seki, D5-brane in Anti-de Sitter Space and Penrose Limit, hep-th/0205266. 